\title{
Numerical entropy production for shallow water flows
}

\author{
Sudi Mungkasi ${ }^{1} \quad$ Stephen G. Roberts ${ }^{2}$
}

(Received 14 December 2010; revised 4 April 2011)

\begin{abstract}
A numerical scheme for the entropy of the one dimensional shallow water wave equations is presented. The scheme follows from a wellbalanced finite volume method for the quantity vector having water height and momentum as its components. The local truncation error of the entropy is called the numerical entropy production, and can be used to detect the location of a shock discontinuity. We show by numerical tests that the numerical entropy production performs better in detecting such a discontinuity than two local truncation errors of the numerical quantity.
\end{abstract}

\section{Contents}

1 Introduction

2 A well-balanced scheme

http://anziamj.austms.org.au/ojs/index.php/ANZIAMJ/article/view/3786 gives this article, (c) Austral. Mathematical Soc. 2011. Published April 9, 2011. ISSN 1446-8735. (Print two pages per sheet of paper.) Copies of this article must not be made otherwise available on the internet; instead link directly to this URL for this article. 
3 Numerical entropy production

4 Numerical tests

C6

4.1 Moving shock in a dam break problem . . . . . . . . . .

4.2 Stationary shock on a parabolic obstruction . . . . . . C C10

4.3 Shock-like detection . . . . . . . . . . . . . . C C12

5 Conclusions

C14

References

C16

\section{Introduction}

A smoothness indicator is always required by an adaptive numerical method used to solve the shallow water wave equations over a given domain. The smoothness indicator is applied to initiate an adaptive action to be undertaken in some parts of the domain. We propose the numerical entropy production as a smoothness indicator or a shock detector for shallow water flows. This new application of the numerical entropy production is adapted from its application to gas dynamics presented by Golay [4] and Puppo [8].

This is the first study to report the application of the numerical entropy production as a smoothness indicator or a shock detector for shallow water flows. The main result of our work is that the numerical entropy production performs better in detecting a shock discontinuity in shallow water flows than the smoothness indicators proposed by Karni, Kurganov and Petrova [5] and Constantin and Kurganov [3].

We consider the one dimensional shallow water wave equations

$$
\mathbf{q}_{\mathrm{t}}+\mathbf{f}(\mathbf{q})_{\mathrm{x}}=\mathbf{s}
$$


in which

$$
\mathbf{q}=\left[\begin{array}{c}
\mathrm{h} \\
\mathrm{hu}
\end{array}\right], \quad \mathbf{f}=\left[\begin{array}{c}
\mathrm{hu} \\
h \mathrm{u}^{2}+\frac{1}{2} g h^{2}
\end{array}\right], \quad \text { and } \quad \mathbf{s}=\left[\begin{array}{c}
0 \\
-g h z_{x}
\end{array}\right],
$$

are respectively the conservative quantity, the flux, and the source term vectors. Here, $x$ represents the one dimensional spatial variable, $t$ represents the time variable, $\mathfrak{u}=\mathfrak{u}(x, t)$ denotes the water velocity, $h=h(x, t)$ denotes the water height (depth), $z=z(x)$ denotes the topography (bed) elevation, $w:=h+z$ is the stage, and $\mathrm{g}$ is the acceleration due to gravity.

\section{A well-balanced scheme}

With appropriate initial and boundary conditions, a finite volume method solves the shallow water wave equations (1) numerically. Suppose at the $\left(i+\frac{1}{2}\right)$ th interface between the $i$ th and $(i+1)$ th cells, we have used a standard technique to reconstruct the values of water depth $h_{i, r}$ and bed $z_{i, r}$ on the right edge of the ith cell and those of water depth $h_{i+1, l}$ and bed $z_{i+1, l}$ on the left edge of the $(i+1)$ th cell.

Following Audusse et al. [1] and Noelle et al. [7], we introduce new reconstructed values of $z$ and $h$ at the $\left(i+\frac{1}{2}\right)$ th interface

$$
\begin{aligned}
z_{i+\frac{1}{2}}^{*} & :=\max \left\{z_{i, r}, z_{i+1, l}\right\}, \\
h_{i, r}^{*} & :=\max \left\{0, h_{i, r}+z_{i, r}-z_{i+\frac{1}{2}}^{*}\right\}, \\
h_{i+1, l}^{*} & :=\max \left\{0, h_{i+1, l}+z_{i+1, l}-z_{i+\frac{1}{2}}^{*}\right\},
\end{aligned}
$$

as the hydrostatic reconstruction, which leads to the auxiliary values $\mathbf{Q}^{*}=$ $\left(h^{*}, h^{*} u\right)$.

A semi-discrete well-balanced finite volume scheme for the conserved quantity $\mathbf{q}$ is

$$
\Delta x_{i} \frac{d}{d t} \mathbf{Q}_{i}+\mathcal{F}^{r}\left(\mathbf{Q}_{i}, \mathbf{Q}_{i+1}, z_{i, r}, z_{i+1, l}\right)-\mathcal{F}^{l}\left(\mathbf{Q}_{i-1}, \mathbf{Q}_{i}, z_{i-1, r}, z_{i, l}\right)=\mathbf{S}_{i}^{(j)},
$$


where the right and left numerical fluxes of the ith cell are

$$
\mathcal{F}^{\mathrm{r}}\left(\mathbf{Q}_{i}, \mathrm{Q}_{\mathrm{i}+1}, z_{\mathrm{i}, \mathrm{r}}, z_{\mathrm{i}+1, \mathrm{l}}\right):=\mathbf{F}\left(\mathbf{Q}_{\mathrm{i}, \mathrm{r}}^{*}, \mathrm{Q}_{\mathrm{i}+1, \mathrm{l}}^{*}\right)+\left[\begin{array}{c}
0 \\
\frac{1}{2} g \mathrm{~h}_{\mathrm{i}, \mathrm{r}}^{2}-\frac{1}{2} \mathrm{~g}\left(\mathrm{~h}_{\mathrm{i}, \mathrm{r}}^{*}\right)^{2}
\end{array}\right],
$$

and

$$
\mathcal{F}^{\mathrm{l}}\left(\mathrm{Q}_{\mathrm{i}-1}, \mathrm{Q}_{\mathrm{i}}, z_{\mathrm{i}-1, \mathrm{l}}, z_{\mathrm{i}, \mathrm{r}}\right):=\mathbf{F}\left(\mathrm{Q}_{\mathrm{i}-1, \mathrm{r}}^{*}, \mathrm{Q}_{\mathrm{i}, \mathrm{l}}^{*}\right)+\left[\begin{array}{c}
0 \\
\frac{1}{2} \mathrm{gh}_{\mathrm{i}, \mathrm{l}}^{2}-\frac{1}{2} \mathrm{~g}\left(\mathrm{~h}_{\mathrm{i}, \mathrm{l}}^{*}\right)^{2}
\end{array}\right],
$$

calculated at $x_{i+1 / 2}$ and $x_{i-1 / 2}$ respectively. Here, $\mathbf{Q}$ is the approximation of the vector $\mathbf{q}$, and $\mathbf{F}$ is a conservative numerical flux consistent with the homogeneous shallow water wave equations computed in such a way that the method is stable. In the numerical simulations presented in Section 4, we take the conservative numerical flux $\mathbf{F}$ due to Kurganov, Noelle, and Petrova [6]. The index $\mathbf{j}$ of $\mathbf{S}_{\mathbf{i}}^{(\mathbf{j})}$ in equation (6) denotes the order of the numerical source term, where a second order numerical source term is

$$
\mathbf{S}_{i}^{(2)}:=\left[\begin{array}{c}
0 \\
\frac{1}{2} g\left(h_{i, l}+h_{i, r}\right)\left(z_{i, l}-z_{i, r}\right)
\end{array}\right] .
$$

Section 3 presents a numerical scheme for the evolution of the entropy $\eta$ and the definition of the numerical entropy production. To apply the numerical entropy production as a smoothness indicator or a shock detector, taking a well-balanced scheme is not a must. However, in this work we choose a well-balanced scheme because it is more robust than a standard scheme in solving the shallow water wave equations, in that it preserves a steady state of 'a lake at rest' [7].

\section{$3 \quad$ Numerical entropy production}

Bouchut [2] showed how to derive an entropy inequality from the shallow water wave equations (1) by simple algebraic operations. The entropy inequality is

$$
\eta_{t}+\psi_{x} \leqslant 0
$$


where

$$
\eta(\mathbf{q})=\frac{1}{2} h u^{2}+\frac{1}{2} g h^{2}+g h z \quad \text { and } \quad \psi(\mathbf{q})=\frac{1}{2} h u^{3}+g h^{2} u+g h z u
$$

are the entropy and the entropy flux for the shallow water wave equations (1). For smooth solutions, which are without discontinuities, the entropy inequality (10) becomes an equality, called the entropy equation. For solutions with discontinuities, the entropy inequality (10) becomes a strict inequality.

The evolution of the entropy $\eta$ together with the evolution of the conserved quantity q can be used to detect the location of shock waves accurately. For the evolution of the entropy $\eta$, we adopt the semi-discrete scheme (6) with the numerical entropy $\Theta$, the numerical entropy fluxes $\Psi^{r}, \Psi^{l}$, and the numerical source 0 in place of the numerical quantity $\mathbf{Q}$, the numerical quantity fluxes $\mathcal{F}^{r}$, $\mathcal{F}^{\mathrm{l}}$, and the numerical source $\mathbf{S}_{\mathfrak{i}}^{(j)}$ respectively. Therefore, given the value of $\Theta_{i}^{n-1}:=\eta\left(\mathbf{Q}_{i}^{n-1}\right)$, we take a semi-discrete scheme

$$
\Delta x_{i} \frac{d}{d t} \Theta_{i}+\Psi^{r}\left(Q_{i}, Q_{i+1}, z_{i, r}, z_{i+1, l}\right)-\Psi^{l}\left(Q_{i-1}, Q_{i}, z_{i-1, r}, z_{i, l}\right)=0
$$

to obtain the value of $\Theta_{i}^{\mathfrak{n}}$, where

$$
\begin{aligned}
\Psi^{\mathrm{r}}\left(\mathrm{Q}_{i}, \mathrm{Q}_{\mathrm{i}+1}, z_{\mathrm{i}, \mathrm{r}}, z_{\mathrm{i}+1, l}\right) & :=\Psi\left(\mathrm{Q}_{\mathrm{i}, \mathrm{r}}^{*}, \mathrm{Q}_{\mathrm{i}+1, \mathrm{l}}^{*}, z_{\mathrm{i}+1 / 2}^{*}\right) \\
\text { and } \quad \Psi^{\mathrm{l}}\left(\mathrm{Q}_{\mathrm{i}-1}, \mathrm{Q}_{\mathrm{i}}, z_{\mathrm{i}-1, \mathrm{l}}, z_{\mathrm{i}, \mathrm{r}}\right) & :=\Psi\left(\mathbf{Q}_{\mathrm{i}-1, \mathrm{r}}^{*}, \mathrm{Q}_{\mathrm{i}, \mathrm{l}}^{*}, z_{\mathrm{i}-1 / 2}^{*}\right)
\end{aligned}
$$

are the right and left numerical entropy fluxes of the ith cell calculated at $x_{i+1 / 2}$ and $x_{i-1 / 2}$ respectively. Here, $\Theta$ is the approximation of the entropy $\eta$, and $\Psi$ is a consistent numerical entropy flux in the sense that $\Psi(\mathbf{Q}, \mathbf{Q})=\Psi(\mathbf{Q})$ (Tadmor [10] gives more details on a consistent numerical entropy flux). As in scheme (6), we use the auxiliary values $\mathbf{Q}^{*}$ instead of the true values $\mathbf{Q}$. At the $\left(i+\frac{1}{2}\right)$ th interface, the transferred entropy is accounted for by heights $h_{i, r}^{*}$ and $h_{i+1, l}^{*}$ together.

The numerical entropy production is then defined by

$$
E_{i}^{n}=\frac{1}{\Delta t}\left[\eta\left(Q_{i}^{n}\right)-\Theta_{i}^{n}\right]
$$


which is the local truncation error of the entropy. Here, $\Delta t$ is the time step used in solving the semi-discrete ODEs (6) and (12).

The order of accuracy for the numerical entropy production (15) in a rough region (nonsmooth solution) is lower than the order of accuracy in a smooth region (smooth solution) [9]. As a result, the values of the numerical entropy production in the region of a shock discontinuity are larger than the values in smooth regions. This property makes the numerical entropy production a good candidate as a smoothness indicator or a shock detector.

\section{$4 \quad$ Numerical tests}

We consider one test involving a moving shock and another test involving a stationary shock in order to judge the performance of the numerical entropy production in detecting shocks. In addition, a test exhibiting a shock-like detection is also considered. We compare the performance of the numerical entropy production (NEP) given by (15) to Karni, Kurganov, and Petrova's (KKP) local truncation error [5] of the mass of water and Constantin and Kurganov's (CK) local truncation error [3] of the mass of water. The KKP indicator is defined at $(x, t)=\left(x_{i}, t^{\mathfrak{n}}\right)$ by

$$
\begin{aligned}
E_{i}^{n}= & \frac{1}{12}\left\{\Delta x\left[h_{i+1}^{n+1}-h_{i+1}^{n-1}+4\left(h_{i}^{n+1}-h_{i}^{n-1}\right)+h_{i-1}^{n+1}-h_{i-1}^{n-1}\right]\right. \\
& +\Delta t\left[h_{i+1}^{n+1} u_{i+1}^{n+1}-h_{i-1}^{n+1} u_{i-1}^{n+1}+4\left(h_{i+1}^{n} u_{i+1}^{n}-h_{i-1}^{n} u_{i-1}^{n}\right)\right. \\
& \left.\left.+h_{i+1}^{n-1} u_{i+1}^{n-1}-h_{i-1}^{n-1} u_{i-1}^{n-1}\right]\right\}
\end{aligned}
$$

whereas the CK indicator is defined at $(x, t)=\left(x_{i+1 / 2}, t^{n-1 / 2}\right)$ by

$$
\begin{aligned}
E_{i+1 / 2}^{n-1 / 2}= & \frac{1}{2}\left\{\Delta x\left[h_{i}^{n}-h_{i}^{n-1}+h_{i+1}^{n}-h_{i+1}^{n-1}\right]\right. \\
& \left.+\Delta t\left[h_{i+1}^{n-1} u_{i+1}^{n-1}-h_{i}^{n-1} u_{i}^{n-1}+h_{i+1}^{n} u_{i+1}^{n}-h_{i}^{n} u_{i}^{n}\right]\right\} .
\end{aligned}
$$

The order of accuracy for the KKP indicator (16) in a rough region is lower than the order of accuracy in a smooth region [5], and the same is true [3] 
for the CK indicator (17). Therefore, the KKP and CK indicators can also be used as smoothness indicators or shock detectors.

In the tests, we use the flux function due to Kurganov et al. [6] for the numerical evolution of the conserved quantity $\mathbf{q}$ and the entropy $\eta$. This flux function is defined for homogeneous conservation laws. Since Kurganov et al. [6] explicitly presented the flux for the numerical evolution of the conserved quantity $\mathbf{q}$, here we write only the flux for the numerical evolution of the entropy $\eta$. The flux for the numerical evolution of the entropy $\eta$ at the $\left(i+\frac{1}{2}\right)$ th interface is

$$
\begin{aligned}
\Psi_{i+\frac{1}{2}}(t)= & \frac{a_{i+\frac{1}{2}}^{+} \psi\left(\mathbf{q}_{i+\frac{1}{2}}^{-}\right)-a_{i+\frac{1}{2}}^{-} \psi\left(\mathbf{q}_{i+\frac{1}{2}}^{+}\right)}{a_{i+\frac{1}{2}}^{+}-a_{i+\frac{1}{2}}^{-}} \\
& +\frac{a_{i+\frac{1}{2}}^{+} a_{i+\frac{1}{2}}^{-}}{a_{i+\frac{1}{2}}^{+}-a_{i+\frac{1}{2}}^{-}}\left[\eta\left(q_{i+\frac{1}{2}}^{+}\right)-\eta\left(q_{i+\frac{1}{2}}^{-}\right)\right]
\end{aligned}
$$

where

$$
\begin{aligned}
& a_{i+\frac{1}{2}}^{+}=\max \left\{u_{i+\frac{1}{2}}^{-}+\sqrt{g\left(h_{i+\frac{1}{2}}^{-}\right)^{*}}, u_{i+\frac{1}{2}}^{+}+\sqrt{g\left(h_{i+\frac{1}{2}}^{+}\right)^{*}}, 0\right\}, \\
& a_{i+\frac{1}{2}}^{-}=\min \left\{u_{i+\frac{1}{2}}^{-}-\sqrt{g\left(h_{i+\frac{1}{2}}^{-}\right)^{*}}, u_{i+\frac{1}{2}}^{+}-\sqrt{g\left(h_{i+\frac{1}{2}}^{+}\right)^{*}}, 0\right\},
\end{aligned}
$$

and the starred variables follow from the hydrostatic reconstruction (3)-(5). In addition,

$$
\mathbf{q}_{i+\frac{1}{2}}^{+}=\mathbf{p}_{i+1}\left(x_{i+\frac{1}{2}}, t\right) \quad \text { and } \quad \mathbf{q}_{i+\frac{1}{2}}^{-}=\mathbf{p}_{i}\left(x_{i+\frac{1}{2}}, t\right)
$$

are the right and the left values at $x=x_{i+1 / 2}$ of a conservative non-oscillatory piecewise polynomial interpolated vector

$$
\tilde{\mathbf{q}}(x, t)=\sum_{i} p_{i}(x, t) X_{i}
$$


reconstructed at each time step based on the previously computed cell averages, $\left\{\mathbf{Q}_{i}^{\mathfrak{n}}(\mathbf{t})\right\}$, where

$$
Q_{i}^{n}(t)=\frac{1}{\Delta x} \int_{x_{i-1 / 2}}^{x_{i+1 / 2}} q\left(x, t^{n}\right) d x .
$$

The set $\left\{\mathbf{p}_{i}(\cdot, t)\right\}$ consist of vectors with polynomials of a given degree as their components, and $X_{i}$ is the characteristic function for the ith cell.

In all our simulations, the numerical setting is as follows. We use the second order source, second order spatial, and second order temporal discretisations. Quantities are measured in SI units. The acceleration due to gravity is taken as $\mathrm{g}=9.81 \mathrm{~m} / \mathrm{s}^{2}$. The minimum fluid height allowed in the flux computation is $h_{\min }=10^{-6} \mathrm{~m}$. The minmod limiter is used for quantity reconstruction. The CFL number is 1.0. The spatial domain is uniformly discretised into 400 cells.

\subsection{Moving shock in a dam break problem}

As the first test problem, we consider the collapse of a reservoir on a horizontal topography

$$
z(x)=0, \quad 0<x<2000
$$

and an initial condition

$$
u(x, 0)=0, \quad w(x, 0)= \begin{cases}0, & \text { if } 0<x<500 \\ 10, & \text { if } 500<x<1500 \\ 5, & \text { if } 1500<x<2000\end{cases}
$$

The simulation illustrates the motion of the water at any point $x$ in the domain and at any time $t>0$ from the initial condition (25). The condition (25) means that two dam walls (at $x=500$ and $x=1500$ ) are given initially.

Figure 1 shows the simulated water surface (stage) at time $t=20$. Also shown in Figure 1 are the corresponding CK, KKP, and NEP indicators. These results indicate the superiority of the numerical entropy production as a shock 


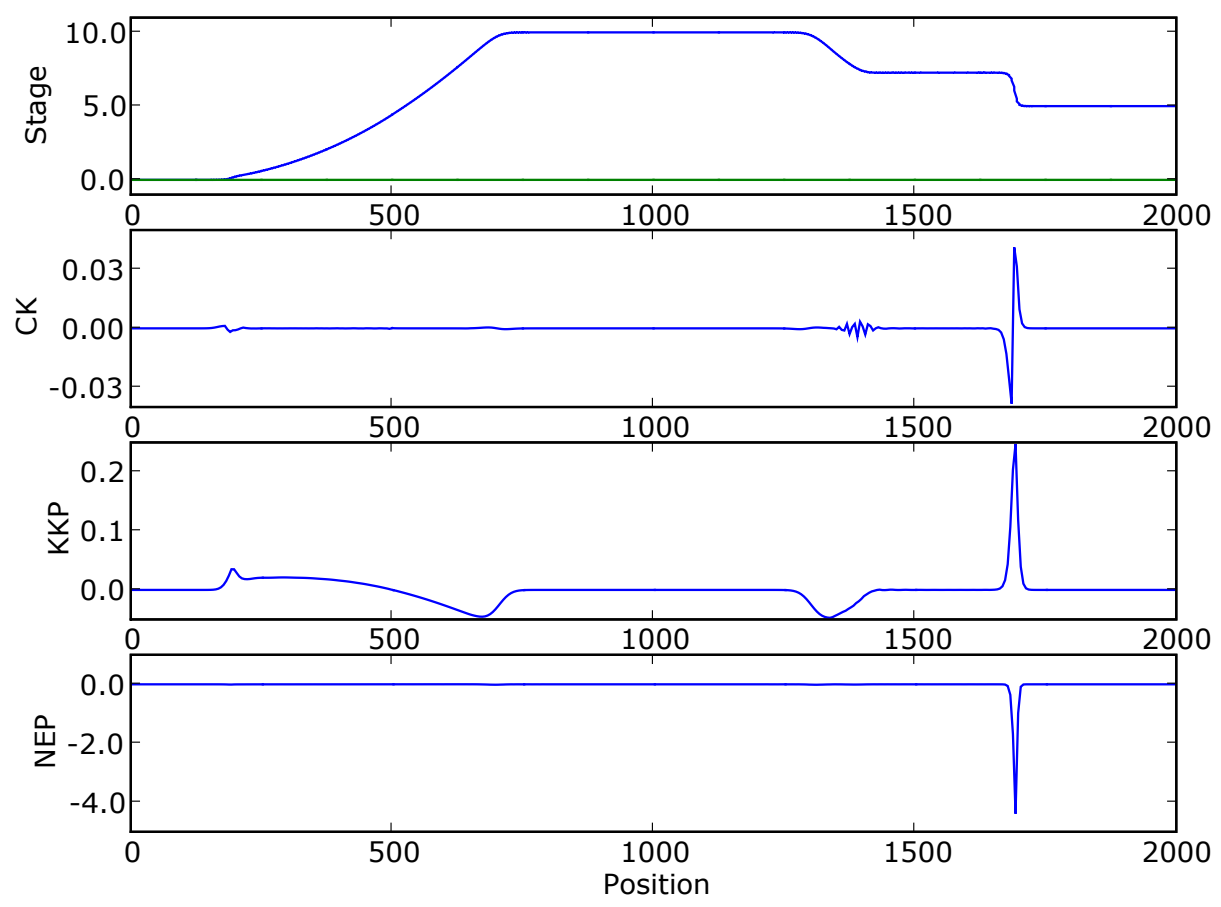

FiguRE 1: A moving shock after dam break at $t=20$. Stage $w$ is the free surface; CK is as in (17); KKP is as in (16); NEP is as in (15). 
detector, when the problem has an unsteady state solution. The magnitude of the NEP is large around the location of the shock and almost zero on the rest of the spatial domain, whereas the CK and the KKP indicators, although large around the location of the shock, also exhibit obvious ripples away from the shock in this test case. Sharp curves of the CK, KKP, and NEP indicators occur only on the region corresponding to the shock, because, as mentioned above, the orders of accuracy of the indicators are lower at the region of the shock than in smooth regions. The supplementary file moving-shock.avi ${ }^{1}$ contains a movie of this first simulation for time $t \in[0,20]$.

\subsection{Stationary shock on a parabolic obstruction}

As the second test problem, we consider a channel of length 25 with topography (having a parabolic bump)

$$
z(x)= \begin{cases}0.2-0.05(x-10)^{2}, & \text { if } 8 \leqslant x \leqslant 12 \\ 0, & \text { otherwise }\end{cases}
$$

and initial condition

$$
u(x, 0)=0, \quad w(x, 0)=0.33,
$$

together with the Dirichlet boundary conditions

$$
\begin{array}{lllll}
{\left[\begin{array}{llllll}
w & h u & z & h & \mathrm{u}
\end{array}\right]=\left[\begin{array}{llllll}
0.42 & 0.18 & 0.0 & 0.42 & 0.18 / 0.42
\end{array}\right]} & \text { at } x=0^{-}, \\
{\left[\begin{array}{llllll}
w & \text { hu } & z & \mathrm{~h} & \mathrm{u}
\end{array}\right]=\left[\begin{array}{lllll}
0.33 & 0.18 & 0.0 & 0.33 & 0.18 / 0.33
\end{array}\right]} & \text { at } \mathrm{x}=25^{+}
\end{array}
$$

Physically, the boundary conditions mean that there is a source of flow upstream quantified by (28) at the point $x=0^{-}$and at the same time there exists a sink of flow downstream quantified by (29) at the point $x=25^{+}$.

${ }^{1}$ http://anziamj .austms.org.au/ojs/index.php/ANZIAMJ/article/ downloadSuppFile/3786/698 

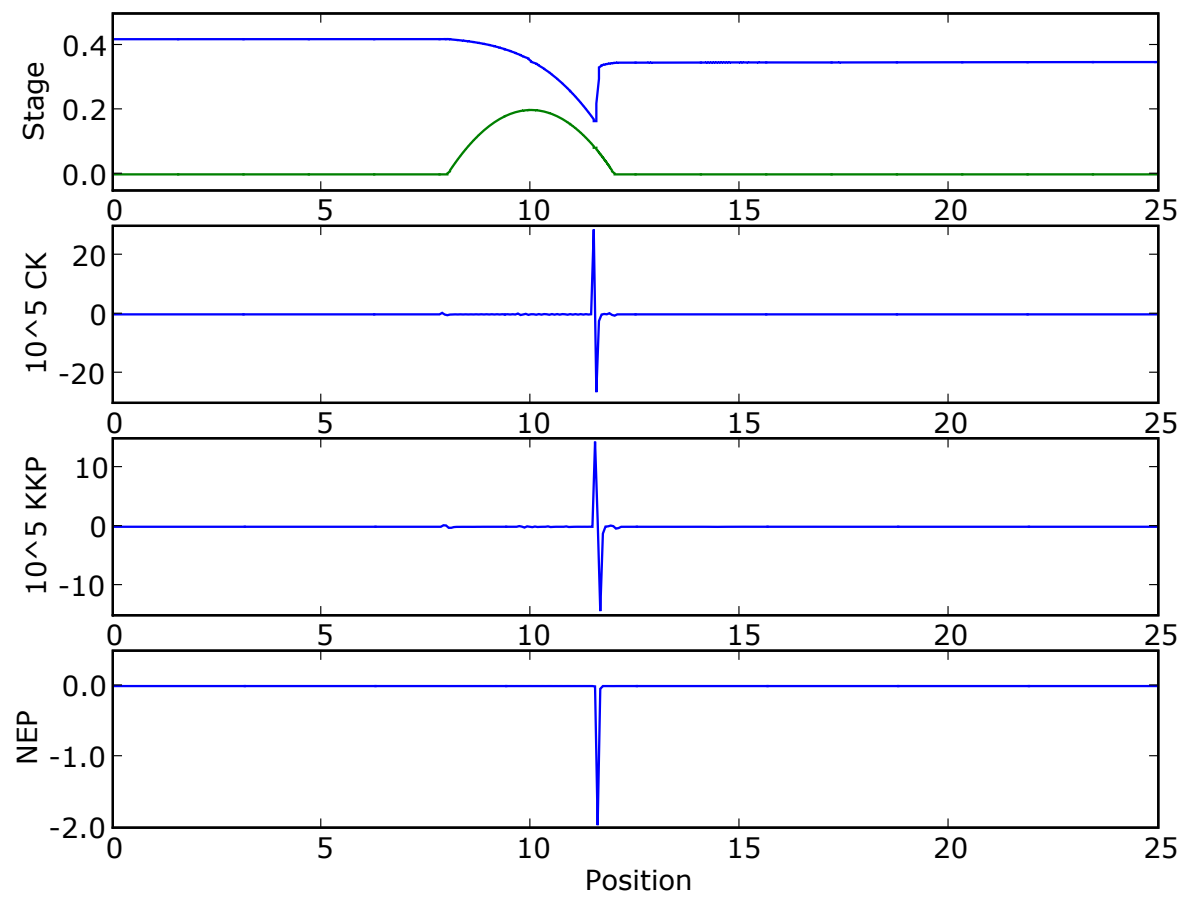

Figure 2: A stationary shock over a parabolic obstruction at $t=50$. Stage $\boldsymbol{w}$ is the free surface; NEP is as in (15). The notation $10^{\wedge} 5 \mathrm{CK}$ and $10^{\wedge} 5 \mathrm{KKP}$ mean $10^{5}$ times $\mathrm{CK}$ and $10^{5}$ times KKP respectively, where CK is as in (17) and KKP is as in (16). 
Figure 2 shows the steady state water surface as well as the corresponding $\mathrm{CK}, \mathrm{KKP}$, and NEP indicators at time $t=50$. These results demonstrate the superiority of the numerical entropy production as a shock detector, when the problem has a steady state solution. The magnitude of the NEP is large around the location of the shock and approximately zero on the rest of the spatial domain, whereas the CK and the KKP indicators, although large around the location of the shock, also exhibit small ripples on the location of the parabolic bump in this test case. The supplementary file steady-shock.avi ${ }^{2}$ contains a movie of this second simulation for time $t \in[0,100]$.

\subsection{Shock-like detection}

Care should be taken in the interpretation of these indicators because false shocks may be detected. For example, in our third test problem we consider the initial condition (27) together with the Dirichlet boundary condition (28) and

$$
\left[\begin{array}{lllll}
w & \text { hu } & z & \mathrm{~h} & \mathrm{u}
\end{array}\right]=\left[\begin{array}{lllll}
0.1 & 0.18 & 0.0 & 0.1 & 0.18 / 0.1
\end{array}\right] \quad \text { at } x=25^{+} .
$$

As in the second test problem, these boundary conditions mean that there is a source of flow upstream quantified by (28) at the point $x=0^{-}$and at the same time there exists a sink of flow downstream quantified by (30) at the point $x=25^{+}$.

In the simulation of this test, a moving shock is detected before the flow is steady. Figure 3 captures the stage as well as the CK, KKP, and NEP indicators at time $t=30$. The shock moves to the right as the time evolves. On the interval where the parabolic obstruction is located, all three smoothness indicators produce some ripples. Those ripples are much smaller in magnitude than the indicator of the moving shock. Once the moving shock has gone from

\footnotetext{
${ }^{2}$ http://anziamj . austms.org.au/ojs/index.php/ANZIAMJ/article/ downloadSuppFile/3786/699
} 

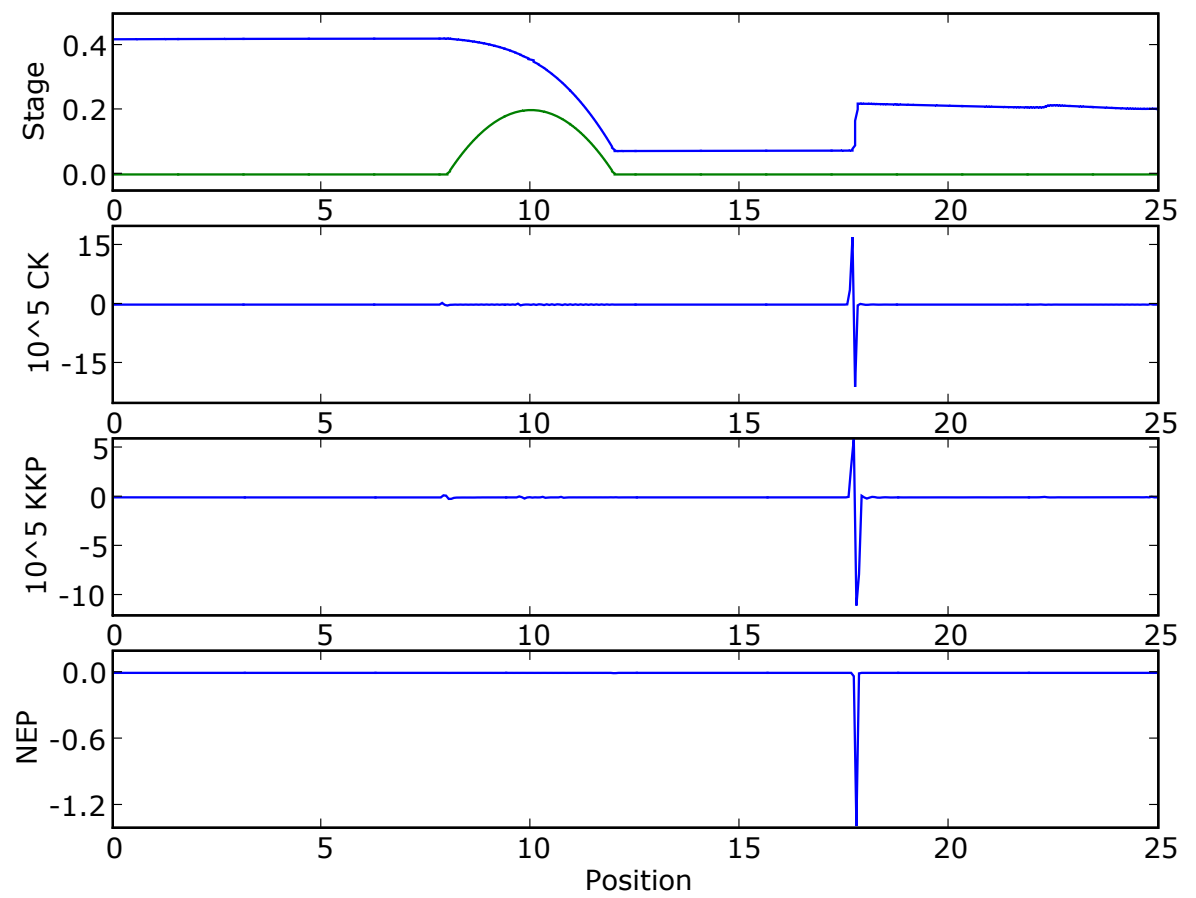

FiguRE 3: A moving shock on the right of a parabolic obstruction at $t=30$. Stage $w$ is the free surface; CK is as in (17); KKP is as in (16); NEP is as in (15). 
the spatial domain of interest, those ripples behave like an indication of a shock since they dominate the values presented by the smoothness indicators.

Figure 4 shows this shock-like behaviour, which captures the stage as well as the CK, KKP, and NEP indicators at time $t=50$. We stress that this shock-like feature is detected with a very small magnitude of indication, which suggests that this detection is actually not a shock. In addition, the NEP performs better because a 'corner' is found on the stage at the corresponding position of the large value of the NEP detector, while the CK and KKP do not detect this 'corner'. The supplementary file shock-like.avi ${ }^{3}$ contains a movie of this third simulation for time $t \in[0,100]$.

\section{Conclusions}

The numerical entropy production provides a good indicator of the smoothness of the water surface profile, and can be used to identify the location of a shock. It performs better than two local truncation errors of the numerical quantity, in that the magnitude of the numerical entropy production is large around the location of a shock and almost zero on the rest of the spatial domain, whereas the two local truncation errors, although large around the location of the shock, also exhibit obvious ripples for continuous solutions. Future research will implement the numerical entropy production as a smoothness indicator for an adaptive finite volume method used to solve the shallow water wave equations.

Acknowledgements We thank Christopher Zoppou at the Mathematical Sciences Institute, The Australian National University (ANU) for some discussions. The work of Sudi Mungkasi was supported by ANU PhD and ANU Tuition Scholarships.

\footnotetext{
${ }^{3}$ http://anziamj . austms . org.au/ojs/index.php/ANZIAMJ/article/ downloadSuppFile/3786/700
} 

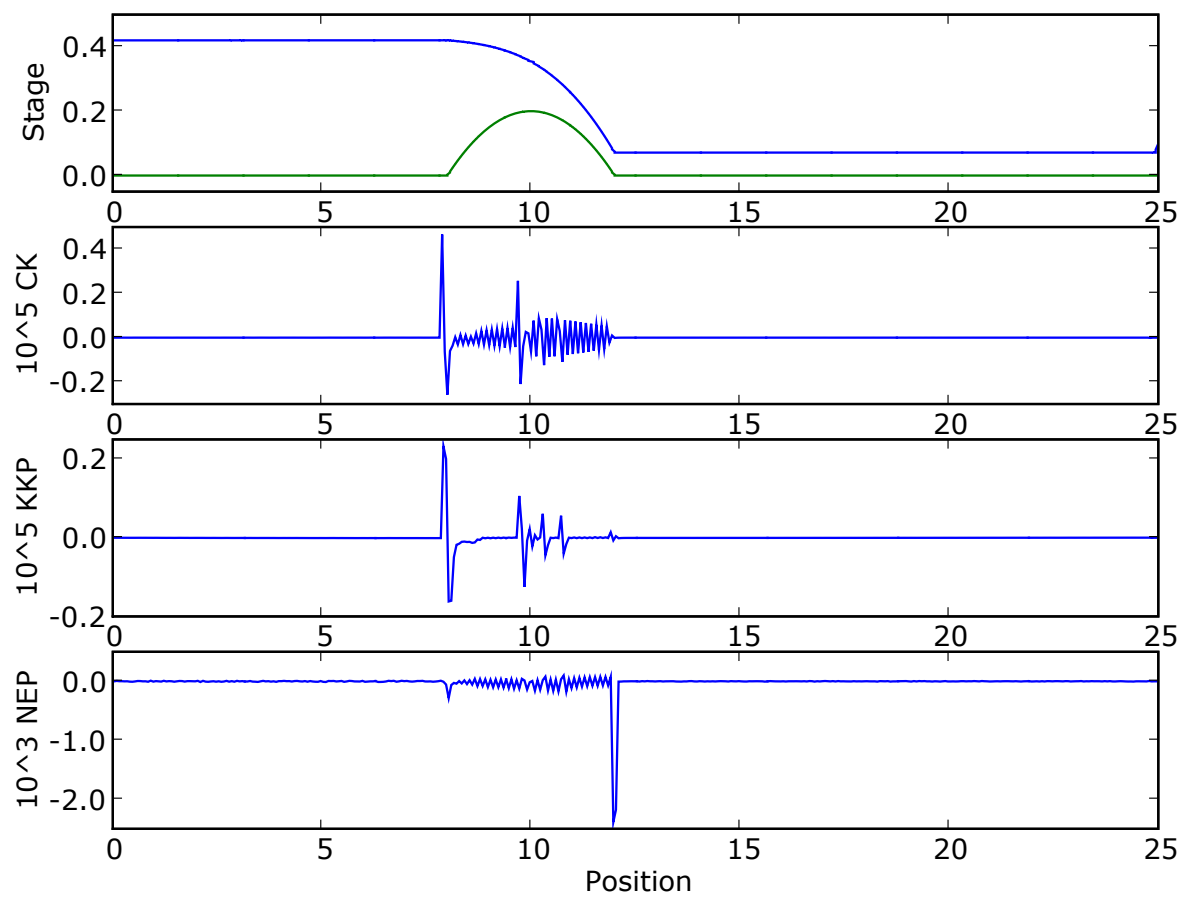

Figure 4: A steady flow without a shock at $t=50$. Stage $w$ is the free surface; CK is as in (17); KKP is as in (16); NEP is as in (15). 


\section{References}

[1] E. Audusse, F. Bouchut, M O. Bristeau, R. Klein, and B. Perthame. A fast and stable well-balanced scheme with hydrostatic reconstruction for shallow water flows. SIAM J. Sci. Comput., 25(6):2050-2065, 2004. doi:10.1137/S1064827503431090 C3

[2] F. Bouchut. Efficient numerical finite volume schemes for shallow water models. In V. Zeitlin, ed., Nonlinear dynamics of rotating shallow water: methods and advances, Vol. 2 of Edited series on advances in nonlinear science and complexity, pages 189-256. Elsevier, 2007. doi:10.1016/S1574-6909(06)02004-1 C4

[3] L A. Constantin, and A. Kurganov. Adaptive central-upwind schemes for hyperbolic systems of conservation laws. In F. Asakura et al., eds., Hyperbolic problems: Theory, numerics, and applications, Vol. 1, pages 95-103. Yokohama Publishers, Yokohama, 2006.

http://129.81.170.14/ kurganov/Constantin-Kurganov.pdf C2, C6

[4] F. Golay. Numerical entropy production and error indicator for compressible flows. C. R. Mecanique, 337(4):233-237, 2009. doi:10.1016/j.crme.2009.04.004 C2

[5] S. Karni, A. Kurganov, and G. Petrova. A smoothness indicator for adaptive algorithms for hyperbolic systems. J. Comput. Phys., 178(2):323-341, 2002. doi:10.1006/jcph.2002.7024 C2, C6

[6] A. Kurganov, S. Noelle, and G. Petrova. Semidiscrete central-upwind schemes for hyperbolic conservation laws and Hamilton-Jacobi equations. SIAM J. Sci. Comput., 23(3):707-740, 2001. doi:10.1137/S1064827500373413 C4, C7

[7] S. Noelle, N. Pankratz, G. Puppo, and J R. Natvig. Well-balanced finite volume schemes of arbitrary order of accuracy for shallow water flows. $J$. 
Comput. Phys., 213(2):474-499, 2006. doi:10.1016/j.jcp.2005.08.019 C3, $\mathrm{C} 4$

[8] G. Puppo. Numerical entropy production on shocks and smooth transitions. J. Sci. Comput., 17(1-4):263-271, 2002. doi:10.1023/A:1015117118157 C2

[9] G. Puppo. Numerical entropy production for central schemes. SIAM J. Sci. Comput., 25(4):1382-1415, 2003 doi:10.1137/S1064827502386712 C6

[10] E. Tadmor. Entropy stability theory for difference approximations of nonlinear conservation laws and related time-dependent problems. Acta Numerica, 12:451-512, 2003. doi:10.1017/S0962492902000156 C5

\section{Author addresses}

1. Sudi Mungkasi, Mathematical Sciences Institute, The Australian National University, Canberra, Australia; Department of Mathematics, Sanata Dharma University, Yogyakarta, InDonesia. mailto: sudi.mungkasi@anu.edu.au, sudi@usd.ac.id

2. Stephen G. Roberts, Mathematical Sciences Institute, The Australian National University, Canberra, Australia. mailto:stephen.roberts@anu.edu.au 